\title{
Teor de Matéria Seca, pH e Amônia Volatilizada da Cama de Frango Tratada ou Não com Diferentes Aditivos
}

\author{
Maria Cristina de Oliveira ${ }^{1}$, Clayton Vieira Almeida ${ }^{2}$, Dalton Oliveira Andrade ${ }^{3}$, \\ Stella Maris Marino Rodrigues ${ }^{4}$
}

\begin{abstract}
RESUMO - O objetivo desse experimento foi determinar o teor de matéria seca, $\mathrm{pH}$ e a amônia volatilizada da cama de frango tratada ou não com diferentes aditivos. Foram utilizadas 440 aves, em delineamento experimental em blocos ao acaso, com cinco tratamentos (1 - cama nova; 2 - cama reutilizada (CR); 3 - CR tratada com sulfato de alumínio; 4 - CR tratada com gesso agrícola e 5 - CR tratada com cal hidratada) e quatro repetições. As amostras da cama foram coletadas quando as aves completaram 42 dias de idade. Não houve influência dos aditivos sobre os resultados de matéria seca, entretanto, a adição de gesso agrícola reduziu o valor de $\mathrm{pH}(6,97)$ e a quantidade de amônia volatilizada $(11,29 \mathrm{mg} / \mathrm{kg})$ da cama de frango. Conclui-se que o gesso agrícola pode ser adicionado à cama, promovendo redução no $\mathrm{pH}$ e na perda de nitrogênio por volatilização da amônia.
\end{abstract}

Palavras-chave: aditivos, amônia, cama de frango, umidade

\section{Dry Matter Content, pH and Volatilized Ammonia from Poultry Litter Treated or Not with Different Additives}

\begin{abstract}
The aim of this experiment was to determine the dry matter content, $\mathrm{pH}$ and the volatilized ammonia from the poultry litter treated or not with different additives. Four hundred and forty birds were used, in randomized block design, with five treatments ( 1 - new litter; 2 - reused litter (RL); 3 - RL treated with aluminum sulfate; 4 - RL treated with gypsum and 5 - RL treated with hydrated lime) and four replicates. The samples of the litter were collected when the birds were 42 days old. There was no influence of the additives on the dry matter results. However, the gypsum reduced the $\mathrm{pH}$ value (6.97) and the quantity of volatilized ammonia (11.29 $\mathrm{mg} / \mathrm{kg}$ ) from the poultry litter. It was concluded that the gypsum can be added to the poultry litter, promoting a reduction in the $\mathrm{pH}$ value and in the losses of nitrogen by ammonia volatilization.
\end{abstract}

Key Words: additives, ammonia, moisture, poultry litter

\section{Introdução}

A amônia é um gás incolor e irritante às mucosas, sendo formado a partir da decomposição microbiana do ácido úrico eliminado pelas aves. Quando a quantidade de amônia inalada é superior a 60 ppm, a ave fica predisposta a doenças respiratórias, aumentando os riscos de infecções secundárias às vacinações. Quando o nível de amônia no ambiente atinge 100 ppm, há redução da taxa e profundidade da respiração, prejudicando os processos fisiológicos de trocas gasosas. Esses níveis altos de amônia (60 a 100 ppm) podem ser observados no início da criação em galpões, com a reutilização da cama (Gonzáles \& Saldanha, 2001).

Os parâmetros mais importantes para se avaliar a qualidade do ar em um galpão são a presença de odores, os níveis de amônia no ar e a concentração de pó, de patógenos no pó e de gás sulfídrico, que podem prejudicar a saúde tanto das aves quanto dos seres humanos (Koerkamp et al., 2000). Pessoas que trabalham com frangos apresentam alta incidência de sintomas agudos e crônicos incluindo tosse, irritação nos olhos, fadiga, congestão nasal, espirro, dor de cabeça, irritação na garganta e febre (Donham, 2000).

$\mathrm{O}$ uso de aditivos na cama de frango é uma solução rápida e econômica para reduzir a volatilização da amônia e amenizar alguns problemas como o aumento na incidência de doenças respiratórias nas aves e no ser humano, a desclassificação de carcaça devido à lesões na pele e também a redução do teor de nitrogênio na cama, o que diminui seu valor como fertilizante.

\footnotetext{
${ }^{1}$ Professora do Departamento de Zootecnia da Fundação do Ensino Superior de Rio Verde (FESURV) - Caixa Postal 166, Rio Verde, GO, CEP: 75.901-970. E.mail: cristina@fesurv.br

2 Estudante de Zootecnia - FESURV. E.mail: vieiraalmeidaclayton@bol.com.br

3 Engenheiro-Agrônomo - Departamento de Agronomia - FESURV.

4 Professora de Química - Departamento de Biologia - FESURV. E.mail: stella@fesurv.br
} 
O sulfato de alumínio é efetivo em reduzir o $\mathrm{pH}$ da cama e, conseqüentemente, a volatilização da amônia. Burgess et al. (1998) compararam o efeito do sulfato de alumínio sobre o $\mathrm{pH}$ da cama de frango composta por palha de arroz e observaram que sua adição provocou redução do $\mathrm{pH}$ de 7,47 para 4,43. Quanto ao teor de matéria seca, os autores relataram não haver efeito da adição desse produto. Ao estudarem os efeitos do sulfato de alumínio sobre o $\mathrm{pH}$, Moore Jr. et al. (2000) verificaram que o sulfato de alumínio reduziu significativamente o $\mathrm{pH}$ da cama, principalmente nas quatro primeiras semanas do ciclo, e a redução do $\mathrm{pH}$ é devido ao fato de o sulfato de alumínio ser um ácido com seis moles de prótons formados para cada mol de sulfato de alumínio dissociado, como apresentado na seguinte equação: $\mathrm{Al}_{2}\left(\mathrm{SO}_{4}\right)_{3} \cdot 14 \mathrm{H}_{2} \mathrm{O}+6 \mathrm{H}_{2} \mathrm{O} \rightarrow 2 \mathrm{Al}(\mathrm{OH})_{3}+3 \mathrm{SO}_{4}^{2-}$ $+6 \mathrm{H}^{+}+14 \mathrm{H}_{2} \mathrm{O}$.

Outro aditivo utilizado para o tratamento de cama de frango é o gesso agrícola $\left(\mathrm{CaSO}_{4}\right)$, que, por intermédio de reações químicas, aumenta a fixação do nitrogênio evitando assim o aumento da concentração de amônia no ambiente (Bruno et al.,1999; Neme et al., 2000). Teuscher \& Adler (1965), citados por Bruno et al. (1999), apresentaram as reações que seriam responsáveis pela fixação da amônia: $\mathrm{CaSO}_{4}+2 \mathrm{NH}_{3}+2 \mathrm{CO}_{2}+2 \mathrm{H}_{2} \mathrm{O} \rightarrow$ $\mathrm{Ca}\left(\mathrm{HCO}_{3}\right)^{2}+\left(\mathrm{NH}_{4}\right) 2 \mathrm{SO}_{4}$.

Wyatt \& Goodman (1992), ao testarem o uso do gesso agrícola como cama de frango ou adicionado a maravalha, observaram que os teores de umidade das camas contendo gesso agrícola foram menores do que o tratamento controle (só maravalha).

A cal hidratada $\left(\mathrm{Ca}(\mathrm{OH})_{2}\right)$ também vem sendo utilizada no intuito de melhorar a qualidade da cama de frango. Este produto foi avaliado com o propósito de reter nitrogênio na cama de frango por Wildey (1984). O autor constatou que a retenção do nitrogênio na cama de frango durou apenas duas semanas, necessitando de nova aplicação após esse período.

Assim, o objetivo deste experimento foi determinar o teor de matéria seca, o $\mathrm{pH}$ e a quantidade de amônia volatilizada de cama de frango não tratada e tratada com diferentes aditivos.

\section{Material e Métodos}

O experimento foi conduzido no Setor de Avicultura da Fundação do Ensino Superior de Rio Verde-FESURV, Goiás, no período de agosto a setembro de 2001.
Foram utilizados 440 pintainhos, com seis dias de idade, ambos os sexos, com peso médio inicial de $93,44 \pm 3,76 \mathrm{~g}$, alojados em galpões de alvenaria divididos em 20 boxes experimentais de $1,85 \mathrm{~m}^{2}$ cada. Adotou-se a densidade de 12 aves $/ \mathrm{m}^{2}$.

O material utilizado como cama em todos os tratamentos foi a casca de arroz, que foi colocada nos boxes sempre na quantidade de $25 \mathrm{~kg}$ por metro quadrado. As camas dos tratamentos 2 a 5 , foram utilizadas anteriormente por um lote de frangos criados em densidade populacional de 12 aves $/ \mathrm{m}^{2}$, durante um período de 42 dias, após o qual passaram por revolvimentos periódicos para que estivessem secas no momento de sua reutilização. $\mathrm{O}$ delineamento experimental foi em blocos ao acaso com cinco tratamentos $(\mathrm{T})$ e quatro repetições. Os tratamentos foram os seguintes: $\mathrm{T} 1$ = cama nova; $\mathrm{T} 2=$ cama reutilizada $(\mathrm{CR})$ sem tratamento; $\mathrm{T} 3=\mathrm{CR}$ tratada com sulfato de alumínio; $\mathrm{T} 4=\mathrm{CR}$ tratada com gesso agrícola e $\mathrm{T} 5=\mathrm{CR}$ tratada com cal hidratada. A aplicação única dos aditivos foi feita na véspera da distribuição das aves nos boxes.

Adicionaram-se 490 gramas de sulfato de alumínio por metro quadrado (Mc Ward \& Taylor, 2000), incorporando-o somente na camada superior da cama. O gesso agrícola foi adicionado na proporção de $40 \%$ do peso da cama, ou seja, foram misturados, no total, $18,5 \mathrm{~kg}$ de gesso agrícola com $27,75 \mathrm{~kg}$ de cama reutilizada (Bruno et al., 1999). A cal hidratada foi utilizada na dosagem de $0,5 \mathrm{~kg} / \mathrm{m}^{2}$ (Colmanetti \& Benedetti, s.d.), sendo incorporada somente na camada superior com um rastelo.

A coleta da cama para as análises foi feita em três pontos diferentes dentro de cada box, evitando-se as áreas próximas e embaixo do comedouro e do bebedouro. Posteriormente, estas três amostras foram homogeneizadas e embaladas.

A determinação do nitrogênio amoniacal foi realizada de acordo com a metodologia descrita por Hernandez et al. (2001) e para matéria seca, utilizouse a técnica de Silva et al. (1991). Para determinação do $\mathrm{pH}$, foram usados $30 \mathrm{~g}$ de amostra, que foram macerados dentro de um béquer. Depois foram adicionados $250 \mathrm{~mL}$ de água deionizada, procedendo-se à agitação desta amostra por cinco minutos. Após, a amostra foi deixada em repouso por 30 minutos antes de se proceder a leitura no $\mathrm{pH}$-metro.

As análises estatísticas foram realizadas pelo método dos quadrados mínimos e a comparação das médias entre os tratamentos foi efetuada pelo teste Duncan.

\footnotetext{
R. Bras. Zootec., v.32, n.4, p.951-954, 2003
} 


\section{Resultados e Discussão}

Os teores de matéria seca, $\mathrm{pH}$ e quantidade de amônia volatilizada encontram-se na Tabela 1.

Não houve diferença $(\mathrm{P}>0,05)$ entre as médias dos tratamentos com relação ao teor de matéria seca, provavelmente, porque a cama dos tratamentos dois a cinco havia sido utilizada anteriormente somente por um lote de frangos e, após a saída das aves, passou por vários revolvimentos e, portanto, tornou-se semelhante à cama do tratamento controle, no que se refere a capacidade de absorver umidade. Sarica \& Çan (1998), ao avaliarem teores de matéria seca de cama de frango reutilizada, obtiveram valores semelhantes (de 67,79 a $69,23 \%$ para diferentes tipos de cama). Autores que utilizaram sulfato de alumínio (Mc Ward \& Taylor, 2000) e gesso agrícola (Neme et al., 2000) também relataram não haver diferença entre tratamentos com ou sem o aditivo.

Houve efeito dos aditivos $(\mathrm{P}<0,05)$ sobre o $\mathrm{pH}$, sendo que o menor valor $(6,97)$ foi obtido na cama de frango tratada com gesso agrícola. Este menor $\mathrm{pH}$ se deve ao fato de o produto ter sido usado em grande quantidade ( $40 \%$ do peso da cama) e de sua alta capacidade de absorver umidade, o que reduz a atividade das bactérias produtoras de amônia, reduzindo assim o $\mathrm{pH}$ da cama. Esperava-se que o sulfato de alumínio provocasse redução do $\mathrm{pH}$, o que não ocorreu. Deve-se ressaltar que a quantidade utilizada foi pequena $\left(490 \mathrm{~g} / \mathrm{m}^{2}\right)$ e misturada somente à porção superior da cama, além disso, a determinação do $\mathrm{pH}$ foi realizada após a saída das aves, quando o produto poderia já ter perdido sua eficiência. Mc Ward \& Taylor (2000) observaram o mesmo efeito. Aos 44 dias, os $\mathrm{pH}$ das camas tratadas com sulfato de alumínio, bissulfato de sódio e argila acidificada eram semelhantes ao da cama sem aditivo (acima de 8,0).

Os resultados foram semelhantes aos obtidos por Moore Jr. et al. (1995) com relação ao uso do sulfato de alumínio, em que os autores utilizaram 100 e $200 \mathrm{~g} / \mathrm{kg}$ de cama e o $\mathrm{pH}$ foi semelhante ao do tratamento controle $(8,89)$ nas duas dosagens $(8,37$ e 7,07 , respectivamente) e aos de Neme et al. (2000), que verificaram redução no $\mathrm{pH}$ das camas com o uso do gesso agrícola. A cal também manteve o $\mathrm{pH}$ elevado, próximo ao do tratamento controle, por ser uma substância alcalina (Colmanetti \& Benedetti, s.d.).

A adição do gesso agrícola, mas não dos outros aditivos, reduziu significativamente $(\mathrm{P}<0,05)$ a quantidade de amônia volatilizada da cama de frango $(11,29 \mathrm{mg} / \mathrm{kg}$ de cama). Este fato ocorreu em virtude de o $\mathrm{pH}$ ter sido mais baixo. $\mathrm{O} \mathrm{pH}$ da cama tem influência direta sobre os níveis de amônia no ar. A volatilização da amônia é baixa, quando o pH é menor que 7 , e aumenta, à medida que o pH se eleva (Reece et al., 1979).

$\mathrm{O}$ pH abaixo de 7 e íons $\mathrm{H}+$ na cama fazem com que aumente a proporção amônio:amônia, ou seja, mais amônia será convertida em íon amônio que não é volátil. A amônia volatiliza, porque não possui carga elétrica (Moore Jr. et al., 2000). Estes resultados diferem daqueles de Neme et al. (2000), os quais

Tabela 1 - Teor de matéria seca, pH e amônia volatilizada da cama de frango tratada ou não com diferentes aditivos Table 1 - Dry matter content, $\mathrm{pH}$ and volatilized ammonia from poultry litter treated or not with dfferent additives

\begin{tabular}{|c|c|c|c|}
\hline \multirow{3}{*}{$\begin{array}{l}\text { Tratamentos } \\
\text { Treatments }\end{array}$} & \multicolumn{3}{|c|}{$\begin{array}{l}\text { Características } \\
\text { Characteristics }\end{array}$} \\
\hline & Matéria seca (\%) & $\mathrm{pH}$ & Amônia volatilizada (mg/kg) \\
\hline & Dry matter (\%) & $p H$ & Volatilized ammonia $(\mathrm{mg} / \mathrm{kg})$ \\
\hline Cama nova & 64,42 & $7,66^{\mathrm{a}}$ & $57,40^{\mathrm{a}}$ \\
\hline New litter & & & \\
\hline Cama reutilizada (CR) & 57,84 & $8,04^{\mathrm{a}}$ & $83,82^{\mathrm{a}}$ \\
\hline Reused litter $(R L)$ & & & \\
\hline $\mathrm{CR}+$ sulfato de alumínio & 61,59 & $7,07^{\mathrm{a}}$ & $32,06^{\mathrm{a}, \mathrm{b}}$ \\
\hline$R L+$ aluminum sulfate & & & \\
\hline $\mathrm{CR}+$ gesso agrícola & 69,07 & $6,97^{\mathrm{b}}$ & $11,29^{b}$ \\
\hline $\begin{array}{l}R L+\text { gypsum } \\
\mathrm{CR}+\text { cal hidratada } \\
R L+\text { hydrated lime }\end{array}$ & 60,00 & $7,85^{\mathrm{a}}$ & $78,10^{\mathrm{a}}$ \\
\hline $\begin{array}{l}\text { Coeficiente de variação }(\%) \\
\text { Coefficient of variation (\%) }\end{array}$ & 9,41 & 4,36 & 25,11 \\
\hline
\end{tabular}

Médias seguidas de letras diferentes, nas colunas, diferem $(P<0,05)$ pelo teste de Duncan.

Means followed by different letters, in columns, differ $(P<.05)$ by Duncan test.

R. Bras. Zootec., v.32, n.4, p.951-954, 2003 
relataram que a adição de gesso agrícola não ajudou na fixação do nitrogênio na cama de frango. Por outro lado, Glória et al. (1991) mencionaram que a adição de gesso, tanto misturado, quanto aplicado só na superfície, promoveu redução na volatilização da amônia (996, 835 e $1065 \mathrm{mg} \mathrm{N} / 200 \mathrm{~g}$ de esterco de galinha, para os tratamentos com gesso misturado, aplicado na superfície e controle, respectivamente). Mc Ward \& Taylor (2000) relataram redução da volatilização da amônia com o uso do sulfato de alumínio, mas, aos 44 dias, já não havia diferença, sendo que cerca de $60 \mathrm{mg} / \mathrm{kg}$ haviam sido volatilizados tanto da cama do tratamento controle como da cama tratada com sulfato de alumínio. Da mesma forma, Stanush et al. (2000) também não observaram diferenças com relação à quantidade de amônia volatilizada com o uso de cal hidratada na cama de frango, sendo que esta quantidade foi determinada aos 49 dias do ciclo.

\section{Conclusões}

O gesso agrícola pode ser adicionado à cama de frango, provocando redução tanto do $\mathrm{pH}$, quanto das perdas de amônia por volatilização.

\section{Literatura Citada}

BRUNO, L.D.G.; MORAES, V.M.B.; ARIKI, J. et al. Efeitos da adição de gesso agrícola à cama aviária sobre o desempenho de frangos de corte. Revista Brasileira de Zootecnia, v.28, n.2, p.320-325, 1999.

BURGESS, R.P.; CAREY, J.B.; SHAFER, D.J. The impact of $\mathrm{pH}$ on nitrogen retention in laboratory analysis of broiler litter. Poultry Science, v.77, n.12, p.1620-1622, 1998.

COLMANETTI, A.L.; BENEDETTI, E. Utilização da cama de frango como fonte alternativa na alimentação de bovinos. In: Viabilidade econômica da cama de frango. Videira: Perdigão, (s.d.). 16p.

DONHAM, K. J. Occupational health hazards and recommended exposure limits for workers in poultry building. In: PROCEEDING NATIONAL POULTRY WASTE MANAGEMENT SYMPOSIUM, 2000, Auburn. Proceedings... Auburn: Auburn University, 2000. p.92-109.

GLÓRIA, N.A.; BARRETTO, M.C.V.; MORAES, C.J. et al. Avaliação do gesso e de alguns fosfatos como inibidores da volatilização de amônia de estercos. Revista Brasileira de Ciência do Solo, v.15, n.3, p.297-301, 1991.
GONZÁLES, E.; SALDANHA, E.S.P.B. Os primeiros dias de vida do frango e a produtividade futura. In: CONGRESSO BRASILEIRO DE ZOOTECNIA, 11., 2001, Goiânia. Anais... Goiânia: AZEG/ABZ, 2001. p.312-313.

HERNANDEZ, R.; GAZETTA, J.O.; MORAES, V.M.B. Método simples e acessível para determinar amônia liberada pela cama aviária. Revista Brasileira de Zootecnia, v.30, n.3, p.824-829, 2001.

KOERKAMP, P. W. G. G.; MIDDELKOOP, J. H. van; ELLEN, H. H. Air quality management and requirements in Europe. In: PROCEEDING NATIONAL POULTRY WASTE MANAGEMENT SYMPOSIUM, 2000, Auburn. Proceedings... Auburn: Auburn University, 2000. p.72-79.

Mc WARD, G.W.; TAYLOR, D.R. Acidified clay litter amendment. Journal of Applied Poultry Research, v.9, n.4, p.518-529, 2000.

MOORE Jr., P.A.; DANIEL. T.C.; EDWARDS, D.R. Reducing phosphorus runoff and inhibiting ammonia loss from poultry manure with aluminum sulfate. Journal of Environmental Quality, v.29, n.1, p.29-37, 2000.

MOORE Jr., P.A.; DANIEL. T.C.; EDWARDS, D.R. et al.Effect of chemical amendments on ammonia volatilization from poultry litter. Journal of Environmental Quality, v.24, n.2, p.293-300, 1995.

NEME, R., SAKOMURA, N.K.; OLIVEIRA, M.S. et al. Adição de gesso agrícola em três tipos de cama de aviário na fixação de nitrogênio e no desempenho de frango de corte. Ciência Rural, v.30, n.4, p.687-692, 2000.

REECE, F.N.; BATES, B.J.; LOTT, B.D. Ammonia control in broiler houses. Poultry Science, v.58, p.754-755, 1979.

SARICA, M.; ÇAM, M. A. The effects of reused litter materials on broiler performances and litter properties. Turkish Journal of Veterinary and Animal Sciences, v.22, n.1, p.213-219, 1998.

SILVA, D.J. Análise de alimentos (métodos químicos e biológicos). Viçosa, MG: Universidade Federal de Viçosa, 1991. p. 116 .

STANUSH, D.D.; BELTRAN, R.; CORSIGLIA, C.M. et al. Effect of hydrated lime on selected litter microflora and poultry growth performance. In: POULTRY SCIENCE ASSOCIATION MEETING, 2000, Montreal. Proceedings... Montreal: ASA, 2000. p.1.

WILDEY, H. Manage turkey litter to control ammonia. Poultry Digest, v.43, p.257, 1984.

WYATT, C. L.; GOODMAN, T. N. Research note: the utilization of recycled sheetrock (refined gypsum) as a litter material for broiler houses. Poultry Science, v.71, p.1572-1576, 1992. 